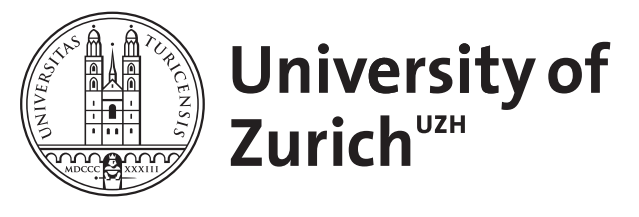

\title{
Hämorrhoidaler Symptomenkomplex
}

\author{
Turina, M ; Slankamenac, K ; Gubler, C
}

\begin{abstract}
Hämorrhoidalleiden gehören mit zu den häufigsten Beschwerden mit denen sich Gastroenterologen, Allgemeinmediziner und Chirurgen konfrontiert sehen. Obschon das Krankheitsbild über die Jahre in seiner Pathologie gut erfasst ist und sich eine Plethora medikamentöser, lokal-interventioneller und letztlich chirurgischer Behandlungsmöglichkeiten ergeben hat, bleibt die Behandlung im Einzelfall oft schwierig. Dieser Leitthemenbeitrag soll einen Überblick über den heutigen Stand der Behandlung hämorrhoidaler Beschwerden vermitteln und Entscheidungshilfe für den Einzelfall bieten. = Hemorrhoids are among the most common complaints with which gastroenterologists, general practitioners and surgeons are faced. Despite the pathology of the disease being well understood and the availability of a range of drugs, locally interventional, and surgical treatment options, the treatment of hemorrhoids can be difficult at times. The purpose of this article is to provide an overview of the current state of treatment for hemorrhoidal complaints and provide decision-making support in individual cases.
\end{abstract}

DOI: https://doi.org/10.1007/s11377-015-0993-9

Posted at the Zurich Open Repository and Archive, University of Zurich

ZORA URL: https://doi.org/10.5167/uzh-207606

Journal Article

Published Version

Originally published at:

Turina, M; Slankamenac, K; Gubler, C (2015). Hämorrhoidaler Symptomenkomplex. Der Gastroenterologe, 10(3):197-202.

DOI: https://doi.org/10.1007/s11377-015-0993-9 
Gastroenterologe $2015 \cdot 10: 197-202$

DOI 10.1007/s11377-015-0993-9

Online publiziert: 3. April 2015

(c) Springer-Verlag Berlin Heidelberg 2015
M. Turina ${ }^{1} \cdot$ K. Slankamenac ${ }^{1} \cdot$ C. Gubler ${ }^{2}$

${ }^{1}$ Klinik für Viszeral- und Transplantationschirurgie, Universitätsspital Zürich

${ }^{2}$ Universitätsspital Zürich

\section{Alte und neue Aspekte}

\section{Hintergrund}

\section{Ätiologie und Epidemiologie}

Der Begriff „Hämorrhoiden“ wird gerne für Missempfindungen im Analbereich synonym verwendet, zu denen Brennen, Juckreiz, Schmerzen beim Stuhlgang oder Blutabgang ab ano gehören. Aufgrund der Häufigkeit dieser Symptome und damit verbundener Arztbesuche sind Aussagen über die exakte Prävalenz schwierig. In einer österreichischen Reihenuntersuchung wurden Hämorrhoiden jedoch bei rund $40 \%$ der Normalbevölkerung festgestellt, wobei nur knapp die Hälfte dieser Gruppe (45\%) Symptome aufwies [1]. Die Bezeichnung „Hämorrhoiden“ indes ist in der medizinischen Terminologie klar definiert: Laut Wikipedia handelt es sich hierbei, einfach formuliert, um „arteriovenöse Gefäßpolster, die ringförmig unter der Analschleimhaut angelegt sind und dem Feinverschluss des Afters dienen“. Letztere Funktion ist im Hinblick auf die Therapie entscheidend, da davon ausgegangen wird, dass rund 15-20\% des Ruheverschlussdrucks durch normalkalibrige Hämorrhoidalpolster geleistet wird [2, 3].

Bezüglich der Entstehung von Hämorrhoidalbeschwerden wird heute in erster Linie von ernährungsbedingten und genetischen Faktoren sowie Stuhlverhaltensmuster ausgegangen. Symptomatische Hämorrhoiden werden mit irregulärem Stuhlverhalten und regelmäßigem Pressen bei Defäkation assoziiert, was entsprechend der Theorie von Thomson letztlich zum Tiefertreten der Hä- morrhoidalpolster führt [4]. Dieses Tiefertreten mit Exposition der Analmukosa außerhalb des Analkanals entsprechend der Stadieneinteilung nach John Goligher (- Tab. 1) erklärt letztlich die typischen Symptome, wie Brennen, Juckreiz, Abgang von Schleim und Blut, und Notwendigkeit zur manuellen Reposition.

Die folgenden Kapitel sollen einen Überblick über die stadiengerechte Therapie von Hämorrhoidalbeschwerden geben. Wichtig erscheint den Autoren hierbei die Erkennung derjeniger Patienten, die früh von einer chirurgischen Behandlung profitieren, andererseits die Identifikation von Patienten, deren Beschwerden mit chirurgischen Maßnahmen (alleine) nicht gebessert werden können und die zunächst einer chirurgischen Maßnahme nicht zugeführt werden sollen. Bei dieser Patientenkategorie stehen nichtoperative Maßnahmen im Vordergrund.

\section{Klinische Beurteilung und Diagnostik}

Die Erstmaßnahme bei Patienten mit hämorrhoidalen Symptomen ist die klinische Sicherung der Diagnose respektive der Ausschluss eines Zweitleidens, das hämorrhoidale (Begleit-)Symptome aufweist. Der Hinweis auf stattgehabten Blutabgang ab ano ist anamnestisch von entscheidender Bedeutung. Schmerzlos, hell und assoziiert mit Stuhlgang ist er mit Hämorrhoidalleiden gut vereinbar, der Abgang von dunklem Blut hingegen meist nicht. Hier muss explizit darauf hingewiesen werden, sich als Kliniker nicht mit dem Vorhandensein von vergrößerten Hämorrhoiden zu begnügen, sondern rechtzeitig die Indikation zur vollständigen Koloskopie zu stellen.

\section{) Die Indikation zur vollständigen Koloskopie ist rechtzeitig zu stellen}

Verbindliche Regeln und Leitlinien existieren nicht, es ist jedoch die Praxis der Autoren, Patienten mit jedweden Warnhinweisen (dunkles Blut, Änderung der Stuhlgewohnheiten, positive Familienanamnese oder persistierende Hämatochezie nach erfolgreicher Hämorrhoidenbehandlung) auch bei jungem Patientenalter einer Koloskopie zuzuführen. Dies gilt insbesondere im Hinblick auf die in mehrfachen Publikationen belegte $\mathrm{Zu}$ nahme der Inzidenz kolorektaler Karzinome bei jugendlichen Patienten [5].

Meist ist die klinische Beurteilung der Hämorrhoiden problemlos möglich, wobei die Autoren ihre Patienten in der lateralen Sims-Position mit angewinkelten Knien untersuchen. Lassen sich proktoskopisch vergrößerte Hämorrhoiden feststellen, kann man mittels Valsalva-Manöver bei entferntem Proktoskop das Ausmaß des Prolapses respektive die Stadieneinteilung nach Goligher ( $\bullet$ Tab. 1) bestimmen. Desweiteren können Blutungsstigmata als Hinweis auf rezidivierende Hämatochezie sowie begleitende proktologische Untersuchungsbefunde, wie eine akute oder chronische Analfissur, perianales Ekzem, oder von Hämorrhoiden 


\begin{tabular}{|ll}
\hline Tab. 1 & Stadieneinteilung der Hämorrhoiden nach Goligher \\
\hline Grad & Definition \\
\hline I & $\begin{array}{l}\text { Hämorrhoiden endoanal vergrößert; kein Prolaps; äußerlich nicht sichtbar; Leitsymptom: } \\
\text { Hämatochezie; Krankheitswert fraglich }\end{array}$ \\
\hline II & $\begin{array}{l}\text { Hämorrhoiden prolabieren beim Pressen aber reponieren danach spontan; gestörte Fein- } \\
\text { kontinenz; Schleimsekretion mit Irritation perianaler Haut (Brennen) }\end{array}$ \\
\hline III & $\begin{array}{l}\text { Hämorrhoiden prolabieren bei Anstrengung und reponieren nicht selbständig, müssen } \\
\text { manuell reponiert werden }\end{array}$ \\
\hline IV & Dauerhafter Prolaps vergrößerter Hämorrhoiden; Reposition auch manuell unmöglich \\
\hline
\end{tabular}

unabhängige Zusatzbefunde, wie Kondylome oder anorektale Polypen, erfasst werden.

\section{Therapie}

\section{Therapieprinzipien}

Entsprechend klinischem Schweregrad und bisheriger Therapie lässt sich bestimmen, welcher Patient primär konservativ behandelt werden kann. Es sind dies Fälle mit erst- und zweitgradigen Hämorrhoiden ohne bisherige spezifische Behandlung, insbesondere bei Vorhandensein einer Obstipation.

\section{》) Stuhlregulatorische Maßnahmen werden generell empfohlen}

Stuhlregulatorische Maßnahmen als generelle Empfehlung beinhalten ausreichend körperliche Bewegung, faserreiche Kost, Quellmittel, wie Flohsamen (z. B. Metamucil $^{\otimes}$ bis 3 gehäufte Kaffeelöffel mit Flüssigkeit), oder später abführende Maßnahmen, wie z. B. macrogolhaltige Präparate. Gerne werden zusätzlich Medikamente zur Tonisierung der Venenwand verschrieben, wie z. B. Diosmin, deren Nutzen jedoch kontrovers diskutiert wird. Wichtig ist vielmehr die primäre Entscheidung zur konservativen Therapie bei erst- und oft auch zweitgradigen Hämorrhoiden bei gering symptomatischen Patienten. Sollten die Symptome zweitgradiger Hämorrhoiden auf diese Maßnahmen nicht oder ungenügend ansprechen, kommen verschiedene ambulant durchführbare Therapien infrage, die keine Narkose benötigen und damit gut auch in der Praxis angewandt werden können.

\section{Ambulante endoskopische Prozeduren}

Die sog. Office-based-Prozeduren werden bewusst ohne Sedation durchgeführt, um die wichtige Rückmeldung betreffend Schmerzhaftigkeit der Intervention direkt während des Eingriffs zu erhalten. Verspürt der Patient bereits beim Ansaugen des Hämorrhoidalkomplexes ins Gerät Schmerzen, ist die Lokalisation zu tief, d. h. zu nah bei der Linea dentata und das Gummiband darf nicht appliziert werden.

Die Eingriffe bedürfen keiner Vorbereitung, es müssen keine Einläufe verabreicht werden. Nach einer kompletten Koloskopie und somit einer Darmreinigung kann das Hämorrhoidalstadium überschätzt werden und die Patienten sind meist noch sediert, sodass eine gleichzeitige Hämorrhoidentherapie wenig sinnvoll oder sogar mit erhöhtem Komplikationsrisiko assoziiert ist.

Mit Intention einer Therapie wird der Analkanal immer starr endoskopiert, die flexible Endoskopie kann diagnostisch eine Hilfe sein. Mit pädiatrischen Koloskopen in geübten Händen ist die Inversionsproktoskopie im Rektum nicht mit einer erhöhten Perforationsgefahr assoziiert und somit diagnostisch erlaubt. Starre Proktoskope, die durchsichtig sind oder eine definierte Öffnung in der Wand zur Therapie besitzen, können einen Vorteil in der Handhabung bieten.

Endoskopische Therapien umfassen folgende Verfahren (geordnet nach Anwendungshäufigkeit): Gummibandligatur, Infrarotkoagulation, Injektionstherapie, bipolare Elektrotherapie, dopplergeführte Laserfotokoagulation, Kryotherapie. Die letzteren 3 Verfahren sind nicht genügend etabliert und werden hier nicht besprochen.

\section{Gummibandligatur}

Diese kann endoskopisch oder aber auf pistolenartigen Applikatoren durch ein Anoskop erfolgen; je nach Gerät sind eines oder mehrere Gummibänder vorgeladen. Die Kappe selbst ist im Lumen hohl, sodass mithilfe von Sog (oder selten einer Zange) das Hämorrhoidalpolster eingesogen werden kann. Die Tiefe der Kappe definiert somit das anzusaugende Volumen. Gelöst werden die Bänder über einen Fadenmechanismus oder mechanisch durch ein feines Gestänge. Das Ansaugen muss schmerzfrei gelingen. Bei Aspiration von kutan innerviertem Analkanal mit Hämorrhoiden wird der Patient sofort über Schmerzen klagen und das Anbringen von Gummibandligaturen ist zu unterlassen.

Üblicherweise werden 1-2 Gummibänder gesetzt, eine größere Zahl führt öfter zu Schmerzen und temporärem Stuhlverhalt. Eine routinemäßige medikamentöse Stuhlregulation mit einem Faserpräparat und die kurzfristige Gabe von nichtsteroidalen Antirheumatika machen Sinn.

\section{》) Üblicherweise werden}

\section{1-2 Gummibänder gesetzt}

Nach 3-7 Tagen fällt das initial ödematöse und dann nekrotische Gewebe ab und es kommt zu einer Ulzeration, die nochmals selbstlimitiert bluten kann; die Narbe entwickelt sich innerhalb von Wochen. Oft werden mehrere Sitzungen benötigt. Das Therapieintervall soll mindestens 6 Wochen betragen, damit die Ulzerationen sicher abgeheilt sind.

\section{Infrarotkoagulation}

Mithilfe von Infrarot wird Hitze kurzzeitig auf das Hämorrhoidalkissen asymmetrisch im Bereiche der zuführenden Gefäße appliziert, um eine Koagulationsnekrose der Gefäße und Submukosa mit letztendlicher Narbe zu erzielen. Entweder wird in den Arbeitskanal des Endoskops eine fiberoptische Probe eingeführt oder aber durch das Anoskop wird mit pistolenartigen Geräten direkt das leicht geknickte Ende auf die zu therapierende Hämorrhoiden gehalten. 


\section{Injektionstherapie}

Unter Sicht, endoskopisch oder durch das Anoskop wird eine Nadel in die Submukosa und nicht in das Gefäß der inneren Hämorrhoide eingeführt und je Hämorrhoide 1-2 ml „sclerosans“ eingespritzt. So wird eine Entzündung induziert, die später zu einer Narbe führen wird. Verschiedene Substanzen werden verwendet: hypertone Kochsalzlösung, hochprozentiger Alkohol, Polidocanol, ölhaltige Phenollösung, Chininlösung u. a. Das heute bevorzugte Polidocanol ist wenig allergen und gleichzeitig lokal anästhesierend.

\section{Komplikationen}

Alle 3 genannten Methoden sind sicher. Leichte Schmerzen treten in bis zu 20\% der Fälle auf; relevante starke Schmerzen in $2 \%$. Schwere Blutungen oder Infektionen bis hin zur Sepsis sind in weniger als $1 \% \mathrm{zu}$ erwarten [6].

\section{Indikation}

Bei kleinen inneren Hämorrhoiden kann die Injektions- oder Infrarottherapie ein gangbarer Weg sein, bei Beiden sind aber hohe Rezidivraten zu erwarten. Die Gummibandligatur ist per se effektiver und bedarf weniger nachfolgender Sitzungen [7]. Bei hoher Blutungsgefahr bei z. B. antikoagulierten Patienten mag die Injektionstherapie bevorzugt werden, da keine Ulzeration, sondern nur eine Thrombosierung und Fibrose induziert wird.

\section{Effizienz}

Alle 3 Verfahren sind bei Hämorrhoiden im Grad I-II in >95\% erfolgreich [8]. Methode der 1. Wahl ist die Gummibandligatur. Bei Grad III ist mit der Gummibandligatur höchstens ein Erfolg von 70\% zu erwarten.

\section{Operative}

\section{Behandlungsmöglichkeiten}

Bei drittgradigen oder großen zweitgradigen Hämorrhoiden, die ungenügend auf die genannten Maßnahmen angesprochen haben, respektive aufgrund ihrer Größe nicht hierzu qualifizieren, kommen die operativen Behandlungen zum Zug. Hier stehen neben den klassischen resezierenden Verfahren verschiedene technische Möglichkeiten zur Auswahl, die nun kurz besprochen werden sollen.

\section{Hämorrhoidalarterienligatur}

Erstmals im Jahr 1995 durch Morinaga et al. [9] beschrieben stellt die Hämorrhoidalarterienligatur (HAL) eine elegante Technik dar, mit der der variable arterielle Zufluss der vergrößerten Hämorrhoiden visuell dargestellt und ligiert werden kann. Später wurde die Technik durch eine Mukopexie, respektive Hämorrhoidopexie, ergänzt, bei der die Mukosa entlang der Hämorrhoidenbasis gerafft wird, um eine zusätzliche Reposition der prolabierten Hämorrhoide nach endoanal zu ermöglichen. Die HAL benötigt ein spezielles Anoskop, das eine entfernbare Dopplersonde enthält, mit der durch Rotieren des Anoskops das zuführende Gefäß dargestellt und über eine Öffnung im Proktoskop kontrolliert durchstochen werden kann.

\section{》) Zur Hämorrhoidalarterien- ligatur wird ein spezielles Anoskop benötigt}

Die Notwendigkeit der dopplergesteuerten Arterienligatur gegenüber der isolierten Mukopexie wird in späteren Studien jedoch kontrovers diskutiert. Es erscheint aber, als ob die Mukopexie alleine einen gleich guten Behandlungseffekt aufweist wie die Kombination aus HAL und Mukopexie [10]. Im Vergleich zur Stapler-Mukosektomie nach Longo und zur klassischen Hämorrhoidektomie konnten weitere Studien einen Vorteil bezüglich Dauer postoperativer Schmerzen aufzeigen, jedoch erscheint die Methode bezüglich der Entwicklung eines operativ behandlungsbedürftigen Rezidivs innerhalb von 24 Monaten unterlegen [11]. Am Patientengut der Autoren spielt diese Technik aus diesem Grund auch eine untergeordnete Bedeutung und die 2 im Folgenden beschriebenen Techniken entsprechen eher der von dem Autoren gelebten täglichen Routine.

\section{Stapler-Mukosektomie nach Longo}

Die Stapler-Mukosektomie, erstmalig im Jahr 1998 von Antonio Longo in Rom vorgestellt, hat aufgrund ihres neuen und
Gastroenterologe 2015 · 10:197-202

DOI 10.1007/s11377-015-0993-9

๑) Springer-Verlag Berlin Heidelberg 2015

\section{Turina $\cdot$ K. Slankamenac $\cdot$ C. Gubler Hämorrhoidaler Symptomenkomplex . Alte und neue Aspekte}

\section{Zusammenfassung}

Hämorrhoidalleiden gehören mit zu den häufigsten Beschwerden mit denen sich Gastroenterologen, Allgemeinmediziner und Chirurgen konfrontiert sehen. Obschon das Krankheitsbild über die Jahre in seiner $\mathrm{Pa}$ thologie gut erfasst ist und sich eine Plethora medikamentöser, lokal-interventioneller und letztlich chirurgischer Behandlungsmöglichkeiten ergeben hat, bleibt die Behandlung im Einzelfall oft schwierig. Dieser Leitthemenbeitrag soll einen Überblick über den heutigen Stand der Behandlung hämorrhoidaler Beschwerden vermitteln und Entscheidungshilfe für den Einzelfall bieten.

\section{Schlüsselwörter}

Epidemiologie - Therapie - Endoskopie ·

Operation - Operationsklammern

\section{Hemorrhoidal disease. Old and new aspects}

\begin{abstract}
Hemorrhoids are among the most common complaints with which gastroenterologists, general practitioners and surgeons are faced. Despite the pathology of the disease being well understood and the availability of a range of drugs, locally interventional, and surgical treatment options, the treatment of hemorrhoids can be difficult at times. The purpose of this article is to provide an overview of the current state of treatment for hemorrhoidal complaints and provide decision-making support in individual cases.
\end{abstract}

Keywords

Epidemiology · Therapy · Endoscopy .

Surgery $\cdot$ Surgical staplers

vielversprechenden Konzepts einen regelrechten Siegeszug in der Hämorrhoidenchirurgie erlebt. Die Technik beruht auf der Entfernung einer zirkulären Manschette, bestehend aus Mukosa und Submukosa, proximal des eigentlichen Hämorrhoidengewebes. Dies bedingt eine Reposition der Hämorrhoide nach anal mit Korrektur des Prolapses ohne schmerzhafte Inzision der $\mathrm{Mu}$ kosa unterhalb der Linea dentata. Hinzu kommt eine zumindest partielle Devas- 


\section{Schwerpunkt}
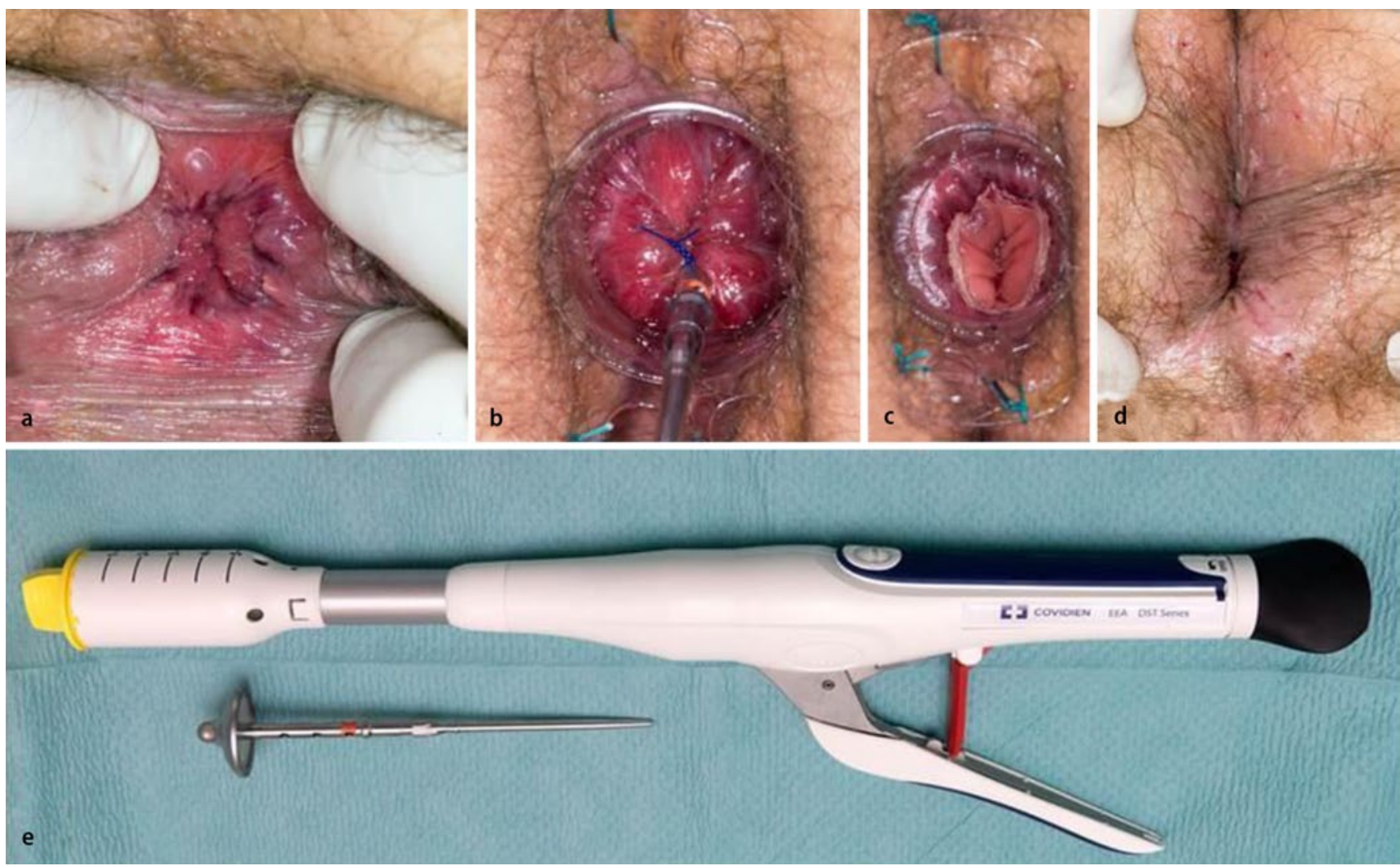

Abb. 1 \ Stapler-Mukosektomie nach Longo. a Zirkuläre zweitgradige Hämorrhoiden. b Knoten der Tabaksbeutelnaht nach Einbringen des Stapler-Kopfs. c Situs nach Auslösen des Staplers. d Finaler Situs von außen. e Covidien EEA ${ }^{\text {TM }}$ Stapler, 33 mm
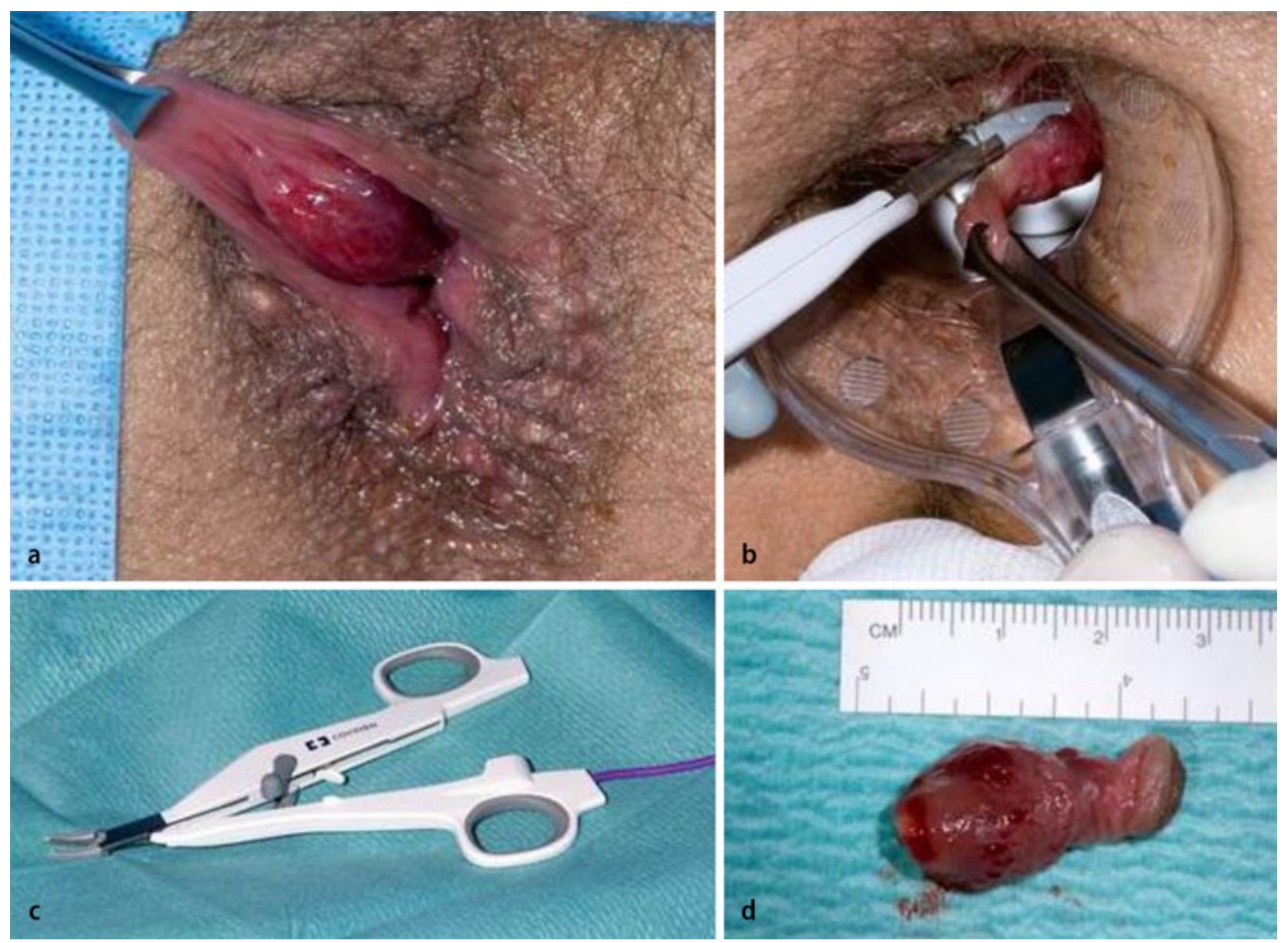

Abb. $2<$ LigaSure ${ }^{\oplus}$-Hämorrhoidektomie. a Isolierte drittgradige Hämorrhoide bei 11 Uhr, Steinschnittlage. b Fassen der Hämorrhoide mit einer Allis-Klemme und Abtragung mittels LigaSure ${ }^{\circledast}$. c LigaSure ${ }^{\circledR}$ Schneidegerät der Fa. Covidien. d Resektat 


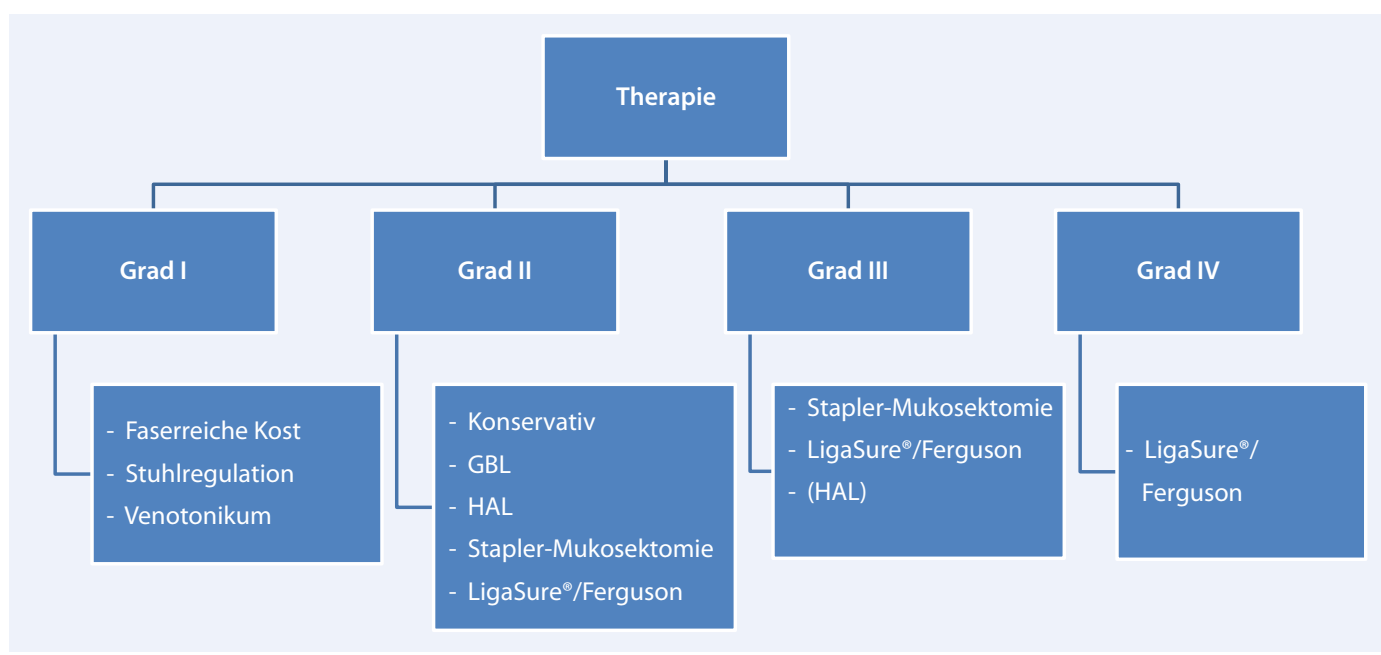

Abb. $3<$ Therapiealgorithmus. Konservativ: identische Therapie wie bei Hämorrhoiden Grad I. GBL Gummibandligatur, $H A L$ Hämorrhoidalarterienligatur

kularisierung der Hämorrhoide, obschon dies nicht das primäre Ziel der Methode darstellt. Gute Indikationen zur Operation nach Longo sind zirkuläre zweitund drittgradige Hämorrhoiden. Viertgradige Hämorrhoiden sind typischerweise mit dieser Technik ungenügend behandelt, da der dauerhaft eingetretene Prolaps nur ungenügend korrigiert werden kann und oft später dennoch eine Hämorrhoidektomie erfolgen muss.

Der operative Ablauf der Stapler-Mukosektomie ist in $\bullet$ Abb. 1 wiedergegeben. Nach Einbringen eines speziellen Nahtanoskops wird eine zirkuläre Tabaksbeutelnaht oberhalb der Hämorrhoidenbasis gelegt, die nach Einbringen des Stapler-Kopfs über diesen geknotet wird. Anschließend wird der Stapler konnektiert, unter Sicht geschlossen und nach Kontrolle der Vagina bei der Frau (Cave: rektovaginale Fistel!) ausgelöst. Das Resektat besteht dann aus dem zirkulären $\mathrm{Ge}$ webering, wobei sich je nach Tiefe der gelegten Tabaksbeutelnaht auch Fasern des Sphinkter ani internus finden können. Auch sonst ist die korrekte Lage der Naht für das Gelingen der Operation entscheidend.

\section{】) Die korrekte Lage der Naht ist für das Gelingen der Stapler- Mukosektomie entscheidend}

Liegt die Naht zu hoch, gelingt die Reposition der Hämorrhoiden nicht genügend, liegt sie zu tief, kommt die Stapel-Reihe in den Bereich der Linea dentata zu liegen und kann zu schwer behandelbaren postoperativen Schmerzen führen.

Die Resultate der Operation nach Longo sind in verschiedenen prospektiven Studien gut beschrieben. Eine randomisierte Studie, in der die Operation mit der klassischen Hämorrhoidektomie nach Ferguson verglichen wurde, wies nach, dass die Stapler-Mukosektomie zu weniger postoperativen Schmerzen und vergleichbaren Resultaten bezüglich Rezidiven nach 12 Monaten führte [12]. Spätere Arbeiten zeigten jedoch bisweilen höhere Raten an Folgeoperationen für rezidivierende Hämorrhoiden mit dieser Operation [13]; das Fazit ist wohl, dass die Stapler-Mukosektomie kurzfristig bezüglich Patientenkomfort bevorzugt wird, sich langfristig die Patienten jedoch möglicherweise häufiger einem Folgeeingriff aufgrund rezidivierter Hämorrhoiden stellen müssen. Am eigenen Patientengut verwenden die Autoren diese Operation routinemäßig für zirkuläre zweitund drittgradige Hämorrhoiden, die mittels Hämorrhoidektomie nicht zufriedenstellend versorgt werden können.

\section{Hämorrhoidektomie nach Ferguson/ LigaSure $^{\oplus}$-Hämorrhoidektomie}

Bei der klassischen Hämorrhoidektomie nach Ferguson werden die betroffene Hämorrhoide und angrenzendes Submukosagewebe bis zur Basis des darunterliegenden Sphinktermuskels abpräpariert, die Hämorrhoide umstochen und abgesetzt und der entstandene Defekt letztlich mit einer Naht partiell verschlossen. Die äu- ßersten $5 \mathrm{~mm}$ der Wunde werden zur Sekretdrainage offen belassen. In den Vereinigen Staaten wahrscheinlich auch heute noch die bevorzugte Technik, stellt die Operation nach Ferguson eine bewährte Behandlungsoption mit guten Resultaten dar. Eine Alternative stellt die „offene“ Hämorrhoidektomie nach Milligan-Morgan dar, bei der die nach Hämorrhoidektomie entstandene Wunde offen belassen wird. Beide Techniken sind in ihren Resultaten ähnlich, wobei die Operationszeit bei der offenen Technik kürzer, aber die Heilungszeit der offenen Wunden länger ist.

Eine interessante und nach Ansicht der Autoren sehr attraktive Alternative zu diesen klassischen resezierenden Techniken stellt die LigaSure ${ }^{\oplus}$-Hämorrhoidektomie dar. Hierbei erfolgt die Präparation der Hämorrhoide mit einem scherenähnlichen Dissektionsgerät, das mittels speziell angelegtem Strom das Gewebe versiegelt und anschließend mit einer mechanisch integrierten Schneidevorrichtung durchtrennt (- Abb. 2). Vorteile dieser Technik sind neben der nochmals deutlich kürzeren und einfacheren Operation eine effektivere Blutstillung sowie geringere postoperative Schmerzen. Die langfristigen Resultate sind den übrigen beschriebenen Techniken zumindestens ebenbürtig, gegenüber der Stapler-Mukosektomie haben mehrere Studien wiederum eine geringere Rezidivrate aufzeigen können [14].

Weitere Varianten der Hämorrhoidektomie sind die rekonstruktive Hämorrhoidektomie nach Fansler-Arnold oder Techniken zur submukösen Gewebedes- 
truktion, wie z. B. die Laserablation oder auch Thermoablation. Obschon jede dieser Techniken ihre Daseinsberechtigung und im Einzelfall ihre Vorteile aufweist, sprengt die genaue Beschreibung dieser Methoden den Rahmen dieses Kapitels.

Bezüglich operativer Maßnahmen bevorzugen die Autoren bei zirkulären zweit- und drittgradigen Hämorrhoiden die Stapler-Mukosektomie nach Longo, bei isolierten Befunden eine Ein- oder Zweizipfelresektion mittels LigaSure ${ }^{\varpi}$. Bei viertgradigen Hämorrhoiden empfehlen die Autoren aufgrund der ihres Erachtens ungenügenden Behandlung mittels Stapler nur resezierende Verfahren, wobei im Einzelfall zur Vermeidung der postoperativen Analstenose auch ein zweizeitiges Vorgehen gewählt werden kann. Hierbei werden bei der Erstoperation die 2 größeren Hämorrhoidenzipfel entfernt und der Patient nach einigen Wochen nachkontrolliert. Sollten dann Restbeschwerden verbleiben, kann nach Ausschluss einer relevanten narbigen Stenose der verbliebene Zipfel gezielt nachreseziert werden. Das Risiko der postoperativen Analstenose ist insgesamt schwer zu beziffern, muss aber insbesondere bei ausgedehntem Befund und resezierendem Verfahren beachtet werden. Vor diesem Hintergrund raten die Autoren von primären Dreizipfelresektionen ab. Der von ihnen propagierte Behandlungsalgorithmus zur Behandlung von Hämorrhoiden ist in - Abb. 3. wiedergegeben.

\section{Fazit für die Praxis}

- Hämorrhoiden sind nach einer österreichischen Reihenuntersuchung bei $40 \%$ der Normalbevölkerung festzustellen. Die klinische Beurteilung der Hämorrhoiden ist meist problemlos z. B in der lateralen Sims-Position mit angewinkelten Knien möglich.

- Therapeutisch stehen neben konservativen Verfahren verschiedene endoskopische und operative Interventionen zur Verfügung.

- Die ambulanten endoskopischen Prozeduren umfassen Gummibandligatur, Infrarotkoagulation und Injektionstherapie. Sie bedürfen keiner Vorbereitung. Unter den operativen Behandlungsmöglichkeiten sind die Hämorrhoidalarterienligatur, die Stapler-Mukosektomie nach Longo und die Hämorrhoidektomie nach Ferguson bzw. die LigaSure ${ }^{\circledR}$-Hämorrhoidektomie zu nennen.

- Die Indikation zur jeweiligen Intervention wird durch den Grad der Erkrankung und den klinischen Befund bestimmt.

\section{Korrespondenzadresse}

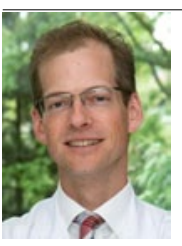

PD Dr. M. Turina

Klinik für Viszeral- und Transplantationschirurgie, Universitätsspital Zürich Rämistr. 100, 8091 Zürich Schweiz matthias.turina@usz.ch

\section{Einhaltung ethischer Richtlinien}

Interessenkonflikt. M. Turina, K. Slankamenac und C. Gubler geben an, dass kein Interessenkonflikt besteht.

Dieser Beitrag beinhaltet keine Studien an Menschen oder Tieren.

\section{Literatur}

1. Riss S, Weiser FA, Schwameis K et al (2012) The prevalence of hemorrhoids in adults. Int J Colorectal Dis 2:215-220

2. Gibbons CP, Trowbridge EA, Bannister JJ, Read NW (1986) Role of anal cushions in maintaining continence. Lancet 8486:886-888

3. Loder PB, Kamm MA, Nicholls RJ, Phillips RK (1994) Haemorrhoids: pathology, pathophysiology and aetiology. Br J Surg 7:946-954

4. Thomson WH (1975) The nature of haemorrhoids. Br J Surg 7:542-552

5. Ahnen DJ, Wade SW, Jones WF et al (2014) The increasing incidence of young-onset colorectal cancer: a call to action. Mayo Clin Proc 2:216-224

6. Davis KG, Pelta AE, Armstrong DN (2007) Combined colonoscopy and three-quadrant hemorrhoidal ligation: 500 consecutive cases. Dis Colon Rectum 9:1445-1449

7. MacRae HM, McLeod RS (1997) Comparison of hemorrhoidal treatments: a meta-analysis. Can J Surg 1:14-17

8. Marques CF, Nahas SC, Nahas CS et al (2006) Early results of the treatment of internal hemorrhoid disease by infrared coagulation and elastic banding: a prospective randomized cross-over trial. Tech Coloproctol 4:312-317

9. Morinaga K, Hasuda K, Ikeda T (1995) A novel therapy for internal hemorrhoids: ligation of the hemorrhoidal artery with a newly devised instrument (Moricorn) in conjunction with a Doppler flowmeter. Am J Gastroenterol 4:610-613
10. Schuurman JP, Borel R, I, Go PM (2012) Hemorrhoidal artery ligation procedure with or without Doppler transducer in grade II and III hemorrhoidal disease: a blinded randomized clinical trial. Ann Surg 5:840-845

11. Elmer SE, Nygren JO, Lenander CE (2013) A randomized trial of transanal hemorrhoidal dearterialization with anopexy compared with open hemorrhoidectomy in the treatment of hemorrhoids. Dis Colon Rectum 4:484-490

12. Senagore AJ, Singer M, Abcarian $\mathrm{H}$ et al (2004) A prospective, randomized, controlled multicenter trial comparing stapled hemorrhoidopexy and Ferguson hemorrhoidectomy: perioperative and oneyear results. Dis Colon Rectum 11:1824-1836

13. Giordano P, Gravante G, Sorge R et al (2009) Longterm outcomes of stapled hemorrhoidopexy vs conventional hemorrhoidectomy: a meta-analysis of randomized controlled trials. Arch Surg 3:266272

14. Chen HL, Woo XB, Cui J et al (2014) Ligasure versus stapled hemorrhoidectomy in the treatment of hemorrhoids: a meta-analysis of randomized control trials. Surg Laparosc Endosc Percutan Tech 4:285289 